\title{
Analysis of Static Bending of Plates FGM Using Refined High Order Shear Deformation Theory
}

\author{
Merdaci $\mathrm{S}^{1,}$, Boutaleb S2, Hellal H², Benyoucef $\mathrm{S}^{2}$
}

1 Structures and Advanced Materials in Civil Engineering and Public Works Laboratory, University of Sidi Bel Abbes, Faculty of Technology, Civil Engineering and Public Works Department, Algeria.

2 Material and Hydrology Laboratory, University of Sidi Bel Abbes, Faculty of Technology, Civil Engineering and Public Works Department, Algeria

* Corresponding Author: slimanem2016@gmail.com

Received: 02-08-2018

Accepted: 04-03-2019

\begin{abstract}
This work deals with the analysis of the mechanical bending behavior of a rectangular plate simply supported on four sides (FGM), subjected to transverse static loading. The high order theory is used in this work, The developed models are variably consistent, have a strong similarity with the classical plate theory in many aspects, do not require correction to the shear factor, and give rise to variations transverse shear stresses such as transverse shear parabolically varies across the shear thickness and satisfies surface conditions without stresses. Equilibrium equations are obtained by applying the principle of virtual works. The mathematical expressions of the arrow, the stresses are obtained using Navies approach to solve the system of equilibrium equations. The influence of mechanical loading and the change of the parameter of the material on mechanical behavior of the plate P-FGM are represented by a numerical example.
\end{abstract}

Key words: FGM, Rectangular plate, Bending, High order theory RPT.

\section{Introduction}

In recent years, functionally graded materials (FGM) have attracted considerable attention in many applications in engineering. Property gradient materials (FGMs), a type of composite material produced by continuously changing the volume fractions in the thickness direction to obtain a definite profile, these types of materials, have received much attention recently in because of the advantages of reducing disparity in material properties and reducing thermal residual stresses (Zhong \& Yu, 2007).

The concept of "Functionally Graded Materials" FGM was developed in Japan National Aerospace Laboratory in 1984 by Mr. Niino and his colleagues in Sendai (Mostefa et al,. 2018). The idea is to produce materials used as thermal barriers in spatial structures and fusion reactors (Koizumi, 1992). The FGM can be used for various applications, such as thermal barrier coatings for ceramic motors, gas turbines, optical thin films, etc. (Nguyen et al., 2008). Due to wide variations and applications of materials with gradient properties, the literature corresponding to the FGMs in the constituents of the materials (Shimpi \& Patel, 2006), fracture mechanics (Wu \& Li, 2010; Şimşek, 2010; Lü et al., 2009; Ying et al., 2009), have been rapidly increased in the past 15 years. Many researchers have devoted their time to understanding the mechanical behavior and mechanism of FGM to provide an optimum profile to designers. Composite plates made of metal and ceramic are widely used in aircraft, ship reactors, and other structural technology applications (Merdaci et al., 2015; Merdaci et al., 2016). Understanding the mechanical behavior of an FGM plate is very important in estimating the safety of the plate (Reddy,. 1984). (Woo \& Meguid, 2001) have applied the Karman theory for the large deformation to obtain the analytical solution for the plates and shells under a mechanical loading transverse and a field of temperature. (Praveen \& Reddy, 1998) studied the static and dynamic responses of thick 
(ceramic-metal) FGM plates using a finite element plate that takes into account the presence of transverse shear stresses, large rotations in the direction of Von Karman. This study deals with the analysis of the mechanical behavior at the static flexion of a rectangular plate of FGM functional gradient materials with the theory of refined shear deformation plates. It is assumed that the material properties of the plate change without interruption across the thickness, depending on the volume fraction of the constituent material according to a power-law function P-FGM. The results obtained show that the mechanical behavior of the rectangular plates FGM is different from those of the isotropic plates. Changing the material parameter has a significant effect on the boom, the normal and tangential stresses.

\section{Refined Plate Theory (RPT)}

An efficient and simple refined plate theory (RPT) was initially introduced and implemented by (Shimpi \& Patel, 2006) in order to deal with the problems of static and dynamic analysis of orthotropic plates. The refined theory can be classified among the third-order shear deformation theories. The development of the refined plate theory is based on the assumptions that the theory represents parabolic variations of shear strains and shear stresses throughout the plate thickness and also satisfies the zero traction boundary conditions on the top and bottom surfaces of the plate. Additionally, the theory can provide high accuracy in prediction plate behavior subjected to mechanical loadings without using the shear correction factor. Based on the basic assumptions of the RPT (Shimpi \& Patel, 2006), the displacement field of the RPT can be written as follows (Merdaci,. 2017):

$$
\begin{gathered}
u(x, y, z)=u_{0}(x, y)-z \frac{\partial w_{b}}{\partial x}+\left(\frac{1}{4} z-\frac{5}{3 h^{2}} z^{3}\right) \frac{\partial w_{s}}{\partial x} \\
v(x, y, z)=v_{0}(x, y)-z \frac{\partial w_{b}}{\partial y}+\left(\frac{1}{4} z-\frac{5}{3 h^{2}} z^{3}\right) \frac{\partial w_{s}}{\partial y} \\
w(x, y, z)=w_{b}(x, y)+w_{s}(x, y)
\end{gathered}
$$

The main differences between the improved TSDT developed by (Shi, 2007) and the RPT are the middle terms of in-plane displacement functions. And the transverse displacement $(\mathrm{w})$ of the RPT is composed of two components of the displacement due to bending (wb) and shear (ws).To apply the improved theory for analyzing plate problems (Merdaci et al,. 2011), it is begun with the Constitutive equations that take the form as,

$$
\left\{\begin{array}{c}
\sigma_{x} \\
\sigma_{y} \\
\tau_{x y}
\end{array}\right\}=\left[\begin{array}{ccc}
Q_{11} & Q_{12} & 0 \\
Q_{12} & Q_{22} & 0 \\
0 & 0 & Q_{66}
\end{array}\right]\left\{\begin{array}{c}
\varepsilon_{x} \\
\varepsilon_{y} \\
\gamma_{x y}
\end{array}\right\} \quad \text { and } \quad\left\{\begin{array}{l}
\tau_{y z} \\
\tau_{z x}
\end{array}\right\}=\left[\begin{array}{cc}
Q_{44} & 0 \\
0 & Q_{55}
\end{array}\right]\left\{\begin{array}{l}
\gamma_{y z} \\
\gamma_{z x}
\end{array}\right\}
$$

$\operatorname{Or}\left(\sigma_{x}, \sigma_{y}, \tau_{x y}, \tau_{y z}, \tau_{y x}\right)$ and $\left(\varepsilon_{x}, \varepsilon_{y}, \gamma_{x y}, \gamma_{y z}, \gamma_{y x}\right)$ are the stress and strain components,

respectively. The stiffness coefficients $Q_{i j}$ are expressed as follows:

$$
Q_{11}=Q_{22}=\frac{E(z)}{1-v^{2}}, \quad Q_{12}=\frac{v E(z)}{1-v^{2}}, \quad Q_{44}=Q_{55}=Q_{66}=\frac{E(z)}{2(1+v)} .
$$

\section{The solution method for FG plate analysis}

The governing equation or the total energy functional based on the improved RPT for FGM plate analysis derived from the energy approach can be solved using the Navier solution in order to determine the static bending results. The total energy functional ( $\Pi$ ) of FGM plates for the static bending analysis can be written as the following,

$$
\Pi=U_{e}+V_{e}
$$


Where $\boldsymbol{U e}$ is the virtual variation of the deformation energy and $\boldsymbol{V e}$ is the variation of work done by external forces can be expressed:

$$
\int_{-h / 2 \Omega}^{h / 2} \int\left[\sigma_{x} \delta \varepsilon_{x}+\sigma_{y} \delta \varepsilon_{y}+\tau_{x y} \delta \gamma_{x y}+\tau_{y z} \delta \gamma_{y z}+\tau_{x z} \delta \gamma_{x z}\right] d \Omega d z-\int_{\Omega}[q \delta w] d \Omega=0
$$

The equilibrium equations can be written from the displacement gradients and put the coefficients $\delta u_{0}, \delta v_{0}, \delta w_{b}$ et $\delta w_{s}$ and equal to zero separately. Thus the equilibrium equations related to the present shear deformation theory can be obtained. The boundary conditions, which are simply supported, are chosen to consider the analysis of the plate.

$$
\begin{aligned}
& v_{0}=w_{b}=w_{s}=\frac{\partial w_{b}}{\partial y}=\frac{\partial w_{s}}{\partial y}=0, N_{x}=M_{x}^{b}=M_{x}^{s}=0 \text { and } x=0, a \\
& u_{0}=w_{b}=w_{s}=\frac{\partial w_{b}}{\partial x}=\frac{\partial w_{s}}{\partial x}=0, N_{y}=M_{y}^{b}=M_{y}^{s}=0 \text { and } y=0, b
\end{aligned}
$$

The assumed in-plane displacements satisfying the conditions of simply supported in direction of $x$ and $y$ are:

$$
\left\{\begin{array}{l}
u_{0} \\
v_{0} \\
w_{b} \\
w_{s}
\end{array}\right\}=\sum_{m=1}^{\infty} \sum_{n=1}^{\infty}\left\{\begin{array}{c}
U_{m n} \cos (\lambda x) \sin \left(\begin{array}{ll}
\mu & y
\end{array}\right) \\
V_{m n} \sin (\lambda x) \cos \left(\begin{array}{ll}
\mu & y
\end{array}\right) \\
W_{b m n} \sin (\lambda x) \sin \left(\begin{array}{ll}
\mu & y
\end{array}\right) \\
W_{s m n} \sin (\lambda x) \sin \left(\begin{array}{ll}
\mu & y
\end{array}\right)
\end{array}\right\}
$$

Where $\boldsymbol{U}_{m n}, \boldsymbol{V}_{\boldsymbol{m} n}, \boldsymbol{W}_{\boldsymbol{b m n}}$ and $\boldsymbol{W}_{\text {smn }}$ are arbitrary parameters to be determined. We obtain the following equation:

$$
[K]\{\Delta\}=\{F\}
$$

Where $\{\Delta\}$ and $\{\boldsymbol{F}\}$ are column vectors

$$
\begin{gathered}
\{\Delta\}^{T}=\left\{U_{m n}, V_{m n}, W_{b m n}, W_{s m n}\right\}, \text { et }\{F\}^{T}=\left\{0,0,-q_{m n},-q_{m n}\right\} . \\
{[K]=\left[\begin{array}{llll}
a_{11} & a_{12} & a_{13} & a_{14} \\
a_{12} & a_{22} & a_{23} & a_{24} \\
a_{13} & a_{23} & a_{33} & a_{34} \\
a_{14} & a_{24} & a_{34} & a_{44}
\end{array}\right]}
\end{gathered}
$$

\section{Results and discussions}

A rectangular ceramic-metal FGM plate according to the P-FGM power-law distribution is considered in this section. The geometry of the FGM plate is shown in figure 1 in which the composition of the material on the upper surface is assumed to be the ceramic-rich surface and the compositions of materials are continuously varied at the metal-rich surface on the opposite side.

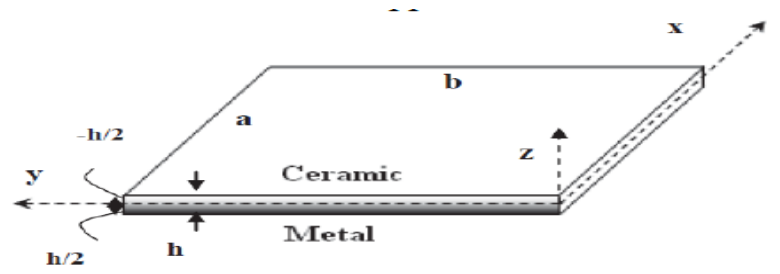

Fig. 1. The dimensions and the variation of the thickness of the rectangular plate FGM. 
In this study, the bending analysis of the FGM plates by the present theory of trigonometric shear deformation is proposed. Comparisons are made with the solutions available in the literature. In order to verify the accuracy of this analysis, some numerical examples are undertaken. The properties of the materials used in the present study are:

Metal (Aluminium,Al): $\boldsymbol{E m}=70 \mathrm{GPa} ; \boldsymbol{v}=0.3$,

Ceramic (Alumina, $\mathrm{Al}_{2} \mathrm{O}_{3}$ ): $\boldsymbol{E} \boldsymbol{c}=380 \mathrm{GPa} \quad \boldsymbol{v}=0.3$. Is taken the ratio a/h=10.

Various non-dimensional parameters used are:

Displacement: $\bar{w}=\frac{10 h^{3} E_{c}}{a^{4} q_{0}} w\left(\frac{a}{2}, \frac{b}{2}\right)$,

Axial stress: $\overline{\boldsymbol{\sigma}}_{x}=\frac{\boldsymbol{h}}{\boldsymbol{a} \boldsymbol{q}_{0}} \sigma_{x}\left(\frac{\boldsymbol{a}}{2}, \frac{\boldsymbol{b}}{2}, \frac{\boldsymbol{h}}{2}\right)$,

Shear stress: ${ }^{\bar{\tau}_{x z}=\frac{\boldsymbol{h}}{a \boldsymbol{q}_{0}} \tau_{x z}\left(0, \frac{\boldsymbol{b}}{2}, 0\right)}$, Coordinate Thickness: $\overline{\boldsymbol{z}}=\boldsymbol{z} / \boldsymbol{h}$.

The FGM plate used is a symmetrical plate and has only two layers of equal thickness, there is no base layer. So, $\boldsymbol{h}_{1}=\boldsymbol{h}_{2}=0$.

In this section, we propose to validate the proposed model through some standard tests known from the literature. It is essentially a question of evaluating its precision performance on the transverse displacements for different elongation ratios. In order to validate our model, an example of static flexion will be studied. The example treats the bending of a simply supported isotropic plate subjected to static loading varying linearly across the thickness. A comparison was made with the results of the method of (Timoshenko \& Gere, 1972), the results of EulerBernoulli and (Zenkour, 2004) will also be introduced in the comparison.

Table 1. Effect of the ratio (a / b) of the elongation on the boom for an isotropic plate subjected to linear loading and $(\mathrm{k}=2)$ for the different theory.

\begin{tabular}{|c|c|c|c|c|}
\hline \multirow{2}{*}{$\mathrm{a} / \mathrm{b}$} & Euler-Bernoulli & [Timoshenko1972] & [Zenkour 2004] & Present study \\
\cline { 2 - 5 } & CPT & FPT & SPT & RPT \\
\hline \hline $1 / 4$ & 0.9832 & 1.0003 & 0.9969 & 0.99694 \\
\hline $1 / 2$ & 0.7103 & 0.7249 & 0.7220 & 0.72204 \\
\hline 1 & 0.2774 & 0.2866 & 0.2847 & 0.28478 \\
\hline 2 & 0.0443 & 0.0480 & 0.0473 & 0.04731 \\
\hline 4 & 0.0038 & 0.0049 & 0.0046 & 0.00469 \\
\hline
\end{tabular}

Table 2. Comparison between the different models of normal stress calculation of an isotropic plate subjected to linear loading.

\begin{tabular}{|c|c|c|c|c|}
\hline \multirow{2}{*}{$\mathrm{z} / \mathrm{h}$} & Euler- Bernoulli & [Timoshenko1972] & [Zenkour2004] & Present study \\
\cline { 2 - 5 } & CPT & FPT & SPT & RPT \\
\hline \hline 0.5 & 1.975764 & 1.975764 & 1.983924 & 1.995501 \\
\hline 0.4 & 1.580611 & 1.580611 & 1.581429 & 1.582588 \\
\hline 0.3 & 1.185458 & 1.185458 & 1.182452 & 1.178187 \\
\hline 0.2 & 0.790305 & 0.790305 & 0.786469 & 0.781029 \\
\hline 0.1 & 0.395152 & 0.395152 & 0.392663 & 0.389131 \\
\hline 0 & 0 & 0 & 0 & 0 \\
\hline-0.1 & 0.395152 & 0.395152 & 0.392663 & 0.389131 \\
\hline-0.2 & 0.790305 & 0.790305 & 0.786469 & 0.781029 \\
\hline-0.3 & 1.185458 & 1.185458 & 1.182452 & 1.178187 \\
\hline-0.4 & 1.580611 & 1.580611 & 1.581429 & 1.582588 \\
\hline-0.5 & 1.975764 & 1.975764 & 1.983924 & 1.995501 \\
\hline
\end{tabular}


The results obtained from the deferent models (Timoshenko \& Gere, 1972; Zenkour, 2004) coincide with those resulting from the present method as illustrated in Tables 1 and 2 . Displacements and normal stresses of an isotropic plate subjected to linear loading.

Table 3. Value of displacements, and the effect of the volume fraction "k" with respect to the rectangular plate simply supported in FGM.

\begin{tabular}{|c|c|c|c|c|c|c|}
\hline \multirow{2}{*}{ Theory } & \multicolumn{6}{|c|}{$\bar{w}$} \\
\cline { 2 - 7 } & $\mathrm{K}=0$ & $\mathrm{~K}=1$ & $\mathrm{~K}=2$ & $\mathrm{~K}=3$ & $\mathrm{~K}=4$ & $\mathrm{~K}=5$ \\
\hline \hline CPT & 0.0737 & 0.1900 & 0.2774 & 0.3277 & 0.3554 & 0.3710 \\
\hline FPT & 0.0779 & 0.1970 & 0.2866 & 0.3385 & 0.3673 & 0.3840 \\
\hline SPT & 0.0779 & 0.1960 & 0.284 & 0.3360 & 0.3645 & 0.3808 \\
\hline Present study (RPT) & 0.0779 & 0.1960 & 0.2847 & 0.3360 & 0.3645 & 0.3808 \\
\hline
\end{tabular}

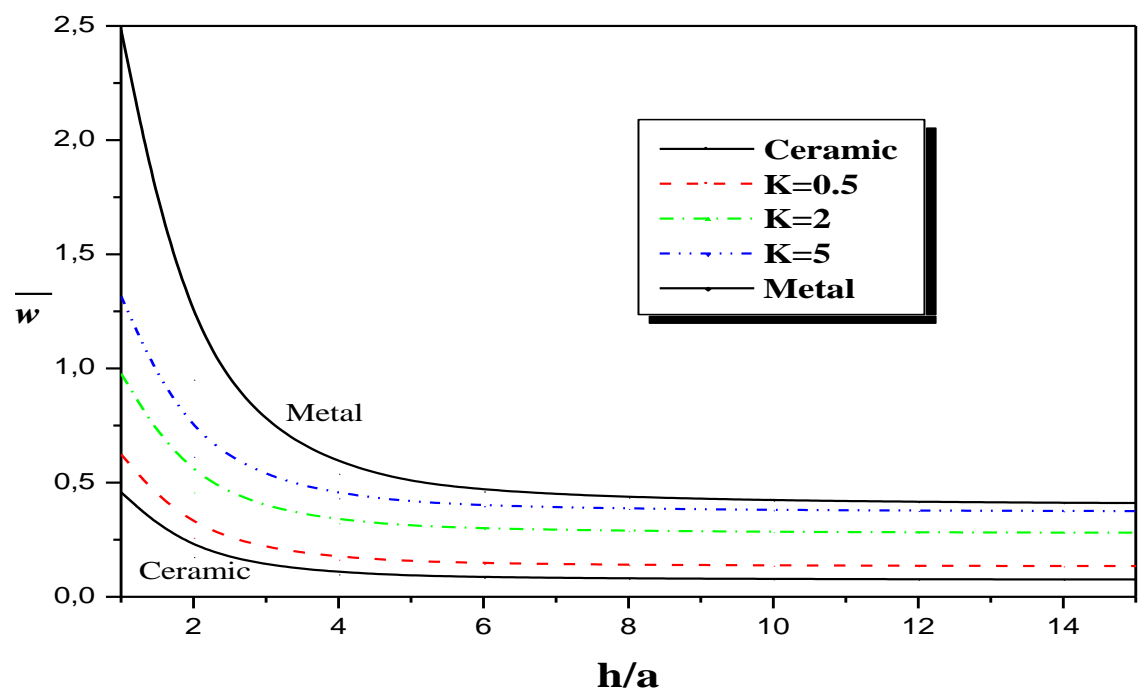

Fig. 2. Influence of thickness (h / a) for various values of "k" of FGM plate.

In the figure 2, shows that the decrease is between those of the ceramic plates $\left(\mathrm{Al}_{2} \mathrm{O}_{3}\right)$ and the metal (Al). It can be observed that the deflection of the metal-rich FGM plate is greater compared to the ceramic plate. This can be taken into account for the Young module.

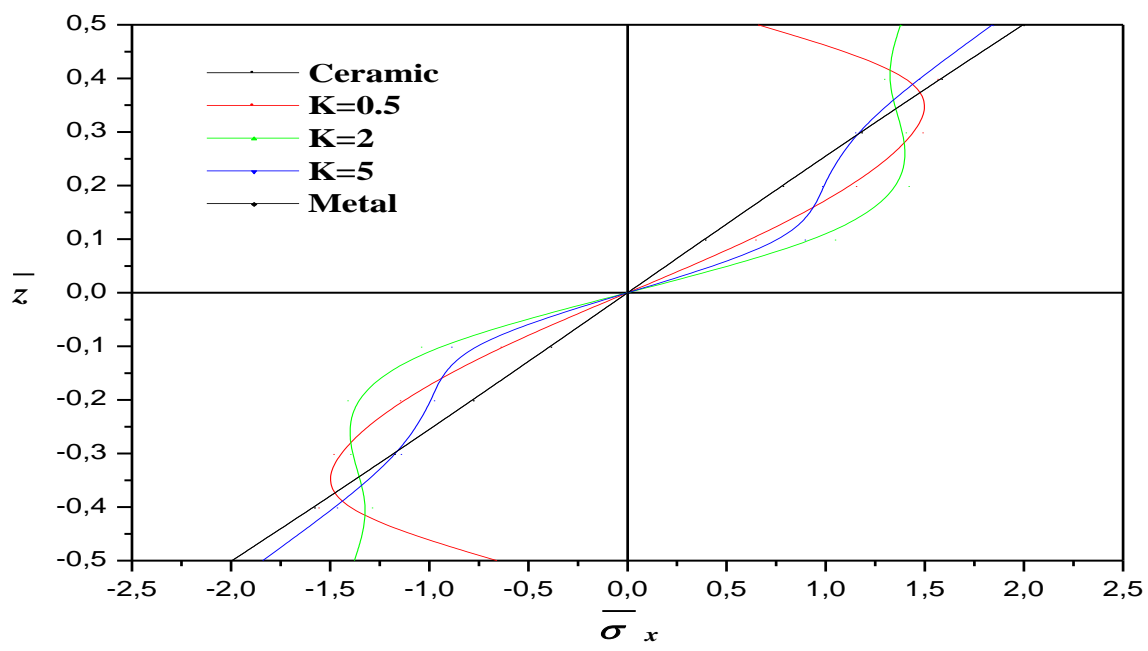

Fig. 3. Variation of the stress, through the thickness of FGM plate. 


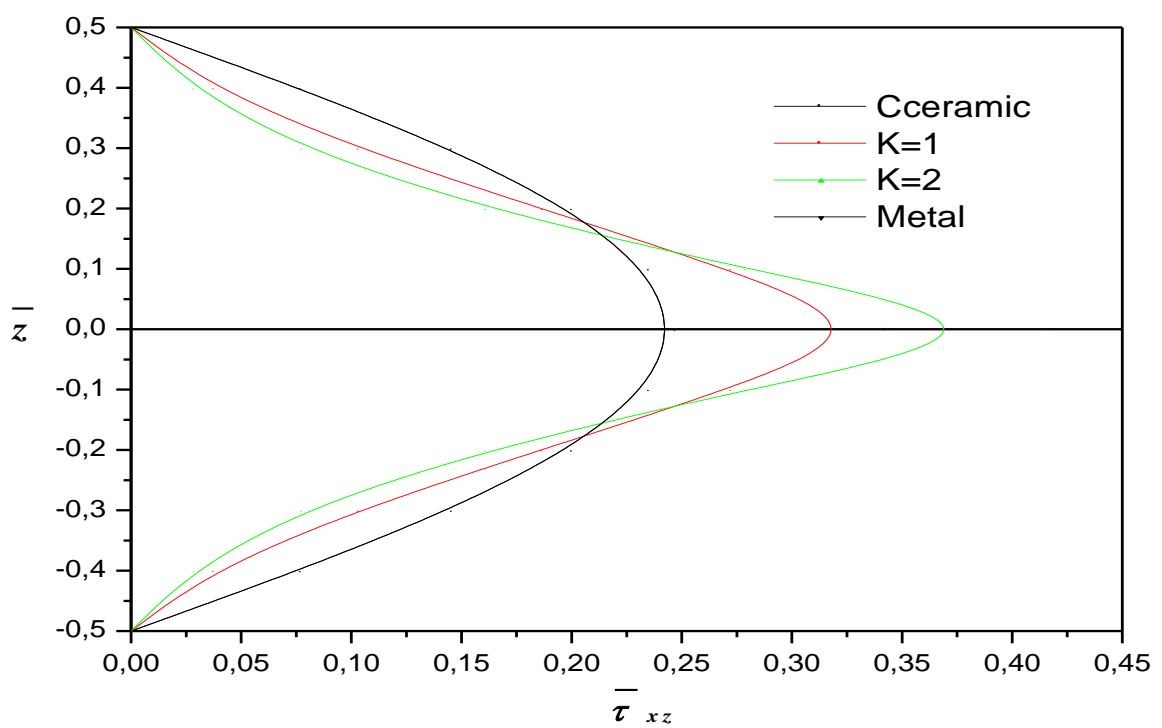

Fig. 4. Transverse shear stress across the thickness of the FGM plate.

In Figure 3, the stresses are tensile above the median plane and under compression below the median plane. The results demonstrate a non-linearity of the variation in the axial stress of sheet thickness for FGM plates. It is important to note that the maximum stress depends on the value of the exponent of the volume fraction " $k$ ". And $n$ figure 4, the maximum value occurs at a point on the median plane of the plate, and its amplitude for an FGM plate is greater than for a homogeneous plate (ceramic or metal).

\section{Conclusion}

This work consists of demonstrating the theoretical plate solutions of FGM functional gradient materials under transverse loading developed using high order theory or refined theory (RPT). The numerical results obtained show that the variation of the modulus of elasticity plays an important role on the distributions of normal and tangential stresses as well as the transverse displacement of the FGM plate. The developed theories give a parabolic distribution of the transverse shear deformation and satisfy the boundary conditions and do not require a shear correction factor as in the case of (FPT). The accuracy and effectiveness of the present theory (RPT) has been demonstrated for the static bending behavior of the FGM plate. All comparative studies have shown that the arrows and stresses obtained using the new four-unknown deformation-shear theory (RPT) are almost identical to those of the other five-unknown theories (SPT). The extension of the current theory is also provided for general boundary conditions and more general form plates. In conclusion, we can say that the proposed (RPT) theory is precise and simple to solve for the study of the behavior of the static bending of rectangular plates in FGM.

\section{References}

Koizumi, M. (1992). Recent progress of functionally gradient materials in Japan. In 16th annual conference on composites and advanced ceramic materials (Vol. 13, p. 333).

Lü, C. F., Lim, C. W., \& Chen, W. Q. (2009). Exact solutions for free vibrations of functionally graded thick plates on elastic foundations. Mechanics of Advanced Materials and Structures, 16(8), 576-584.

Merdaci, S. (2017). Study and Comparison of Different Plate Theory. International Journal of Engineering Research and Advanced Technology 3(8), 48-59. 
Merdaci, S., Belghoul, H., Benyoucef, S., Tounsi, A., Adda Bedia, E.A. (2016). Étude à la Flexion Statique des Plaques Symétriques en FGM Functionally Graded Materials. 9 ème édition des Journées d'Etudes Techniques JET'2016, Hammamet, Tunisie.

Merdaci, S., Benyoucef, S., Tounsi, A., Adda Bedia, E.A. (2015). Analyse Statique des Plaques Homogènes en Matériaux à Gradient Variable (FGM) , 2 ${ }^{\text {nd }}$ International Conference on Aeronautical Sciences I.C.A.S'02 , Oran, Algeria .

Merdaci, S., Tounsi, A., Houari, M. S. A., Mechab, I., Hebali, H., \& Benyoucef, S. (2011). Two new refined shear displacement models for functionally graded sandwich plates. Archive of Applied Mechanics, 81(11), 1507-1522.

Mostefa, A. H., Merdaci, S., \& Mahmoudi, N. (2018). An Overview of Functionally Graded Materials «FGM». In International Symposium on Materials and Sustainable Development (pp. 267-278). Springer.

Nguyen, T. K., Sab, K., \& Bonnet, G. (2008). First-order shear deformation plate models for functionally graded materials. Composite Structures, 83(1), 25-36.

Praveen, G. N., \& Reddy, J. N. (1998). Nonlinear transient thermoelastic analysis of functionally graded ceramic-metal plates. International Journal of Solids and Structures, 35(33), 4457-4476.

Reddy, J. N. (1984). A refined nonlinear theory of plates with transverse shear deformation. International Journal of solids and structures, 20(9-10), 881-896.

Reddy, J. N. (1984). A simple higher-order theory for laminated composite plates. Journal of applied mechanics, 51(4), 745-752.

Shi, G. (2007). A new simple third-order shear deformation theory of plates. International Journal of Solids and Structures, 44(13), 4399-4417.

Shimpi, R. P., \& Patel, H. G. (2006). A two variable refined plate theory for orthotropic plate analysis. International Journal of Solids and Structures, 43(22-23), 6783-6799.

Şimşek, M. (2010). Fundamental frequency analysis of functionally graded beams by using different higher-order beam theories. Nuclear Engineering and Design, 240(4), 697-705.

Timoshenko, S. P., \& Gere, J. M. (1972). Mechanics of Materials. van Nordstrand Reinhold Company. New York.

Woo, J., \& Meguid, S. A. (2001). Nonlinear analysis of functionally graded plates and shallow shells. International Journal of Solids and structures, 38(42-43), 7409-7421.

Wu, C. P., \& Li, H. Y. (2010). An RMVT-based third-order shear deformation theory of multilayered functionally graded material plates. Composite Structures, 92(10), 2591-2605.

Ying, J., Lü, C. F., \& Lim, C. W. (2009). 3D thermoelasticity solutions for functionally graded thick plates. Journal of Zhejiang University-SCIENCE A, 10(3), 327-336.

Zenkour, A. M. (2004). Thermal effects on the bending response of fiber-reinforced viscoelastic composite plates using a sinusoidal shear deformation theory. Acta Mechanica, 171(3-4), 171-187.

Zhong, Z., \& Yu, T. (2007). Analytical solution of a cantilever functionally graded beam. Composites Science and Technology, 67(3-4), 481-488. 